\title{
Partial actions of groups on algebras, a survey
}

\author{
Miguel Ferrero ${ }^{1}$ \\ Instituto de Matemática, Universidade Federal do Rio Grande do Sul \\ 91509-900, Porto Alegre, Brazil. \\ E-mail address: mferrero@mat.ufrgs.br
}

\begin{abstract}
In this survey paper we define partial actions of groups on algebras and give several related results. For any partial action a partial skew group ring is defined. This partial skew group ring is not always associative. Conditions under which associativity holds are studied. Several other questions are considered like, for example, enveloping actions and properties of partial skew group rings.
\end{abstract}

\section{Introduction}

As it is well-known, a group $G$ acts on a set $X$ if for any $g \in G$ there exists a bijection $\alpha_{g}$ of $X$ such that $\alpha_{g} \circ \alpha_{h}=\alpha_{g h}$, for any $g, h \in G$, and $\alpha_{1}=i d_{R}$, where 1 denotes the identity element of $G$. In the case in which $R$ is a $k$-algebra, we say that $G$ acts on $R$ if the above holds and, in addition, any $\alpha_{g}$ is an automorphism of $R$.

A generalization of the above, called a partial action of a group, appeared independently in various areas of mathematics, in particular, in the theory of operator algebras as a powerful tool in their study (see [1], [7], [8], [9], [16], [18]). In the more general setting of a partial action of a group $G$ on a set $X$ the definition is as follows:

Definition Let $G$ be a group with identity element 1 and $X$ be a set. A partial action $\alpha$ of $G$ on $X$ is a collection of subsets $D_{g} \subseteq X(g \in G)$ and bijections $\alpha_{g}: D_{g^{-1}} \rightarrow D_{g}$ such that

(i) $D_{1}=X$ and $\alpha_{1}$ is the identity map of $X$;

\footnotetext{
${ }^{0}$ These notes are based on several recent papers on the subject.

1 Partially supported by Conselho Nacional de Desenvolvimento Científico e Tecnológico (CNPq., Brasil).
} 
(ii) $D_{(g h)^{-1}} \supseteq \alpha_{h^{-1}}\left(D_{h} \cap D_{g^{-1}}\right)$;

(iii) $\alpha_{g} \circ \alpha_{h}(x)=\alpha_{g h}(x)$, for any $x \in \alpha_{h^{-1}}\left(D_{h} \cap D_{g^{-1}}\right)$.

Note that conditions (ii) and (iii) mean that the mapping $\alpha_{g h}$ is an extension of $\alpha_{g} \circ \alpha_{h}$. It is easy to see that condition (ii) can be replaced by the apparently stronger condition: $\alpha_{h^{-1}}\left(D_{h} \cap D_{g^{-1}}\right)=D_{h^{-1}} \cap D_{h^{-1} g^{-1}}$. So, conditions (i), (ii) and (iii) are equivalent to the following:

(i') $D_{1}=X$ and $\alpha_{1}$ is the identity map on $X$;

(ii') $\alpha_{g}\left(D_{g^{-1}} \cap D_{h}\right)=D_{g} \cap D_{g h}$;

(iii') $\alpha_{g}\left(\alpha_{h}(x)\right)=\alpha_{g h}(x)$, for all $x \in D_{h^{-1}} \cap D_{(g h)^{-1}}$.

Throughout these notes $R$ will be always an associative algebra with identity element over a commutative ring $k$. In this case we require that $\left\{D_{g}: g \in G\right\}$ be a family of $k$-algebra ideals of $R$ and every map $\alpha_{g}$ : $D_{g^{-1}} \rightarrow D_{g}$ is an isomorphism of algebras (not necessarily with identities). This definition in a pure algebraic context has been given in [4].

\section{Partial skew group ring}

Let $R$ be an algebra over a commutative ring $k$ and $\alpha=\left\{\alpha_{g}: D_{g^{-1}} \rightarrow\right.$ $\left.D_{g} \mid g \in G\right\}$ a partial action of $G$ on $R$.

1.1. Definition The partial skew group ring $R *_{\alpha} G$ is defined as the set of all finite formal sums $\sum_{g \in G} a_{g} u_{g}, a_{g} \in D_{g}$, with the usual addition and multiplication determined by $\left(a_{g} u_{g}\right)\left(b_{h} u_{h}\right)=\alpha_{g}\left(\alpha_{g}^{-1}\left(a_{g}\right) b_{h}\right) u_{g h}$.

It is easy to see, using the conditions of the definition of partial actions, that the multiplication in $R *_{\alpha} G$ is well defined and defines an structure of algebra over $k$. However this algebra is not always associative.

1.2. Example (cf. [4], Example 3.5) Let $R$ be a four dimensional $K$-vector space (K a field) with basis $\{1, t, u, v\}$. Define the multiplication on $R$ by $u^{2}=v^{2}=u v=v u=t u=u t=t^{2}=0$ and $t v=v t=u$, where 1 is the identity of $R$. Let $\mathrm{G}$ be the cyclic group of order 2 generated by $g$ and let $I$ be the ideal generated by $v$ (i.e., the subspace generated by $u$ and $v$ ). Then the mapping $\alpha_{g}$ given on $D_{g}=I$ by $u \mapsto v$ and $v \mapsto u$ defines a partial action on $R$. It is not difficult to check that $R \star_{\alpha} G$ is not associative. 
So the first question which naturally appears is to find conditions for the associativity of the partial skew group ring.

Obviously the mapping from $R$ to $R *_{\alpha} G$ which sends $a \in R$ to $a u_{1} \in$ $R *_{\alpha} G$ is an embedding and allows us to identify $R$ with $R u_{1} \subseteq R *_{\alpha} G$.

To study when the partial skew group ring is associative the authors in [4] introduced the following notion.

\section{The algebra of multipliers}

Let $R$ be a $k$-algebra with identity element and $I$ an ideal of $R$. Take an element $x \in R$ and consider the left and right multiplication of $I$ by $x: L_{x}: I \rightarrow I, L_{x}(a)=x a$ and $R_{x}: I \rightarrow I, R_{x}(a)=a x$. Then these maps $l=L_{x}$ and $r=R_{x}$ are linear transformations of $I$ which satisfy the following properties, for all $a, b \in I$ :

(a) $l(a b)=l(a) b$;

(b) $r(a b)=\operatorname{ar}(b)$;

(c) $r(a) b=a l(b)$.

2.1. Definition The algebra of multipliers (see [12], [14, 3.12.2] and [4], Section 2) of and algebra $I$ is the set $M(I)$ of all ordered pairs $(l, r)$, where $l$ and $r$ are linear transformations of I which satisfy the properties $(a),(b)$ and $(c)$ above. For $(l, r)$ and $\left(l^{\prime}, r^{\prime}\right)$ in $M(I)$, and $\gamma \in k$ the operations are given by

$\gamma(l, r)=(\gamma l, \gamma r),(l, r)+\left(l^{\prime}, r^{\prime}\right)=\left(l+l^{\prime}, r+r^{\prime}\right)$ and $(l, r)\left(l^{\prime}, r^{\prime}\right)=(l \circ$ $\left.l^{\prime}, r^{\prime} \circ r\right)$.

It is immediate that $M(I)$ is an algebra with identity. Also, the map $\Phi: I \rightarrow M(I)$ defined by $\Phi(x)=\left(L_{x}, R_{x}\right), x \in I$, is a homomorphism of $k$-algebras.

2.2. Definition ([4], Definition 2.4) An algebra $I$ is said to be $(l, r)$ associative if, given two multipliers $(l, r)$ and $\left(l^{\prime}, r^{\prime}\right)$ in $M(I)$, one has that $r^{\prime} \circ l=l \circ r^{\prime}$.

The main result concerning with the associativity question proved in [4] (Theorem 3.1) is the following

2.3. Theorem If $R$ is an algebra and $\alpha$ is a partial action of $G$ on $R$ such that each $D_{g}, g \in G$, is $(l, r)$-associative, then the skew group ring $R \star_{\alpha} G$ is associative. 
Recall that $I$ is said to be idempotent if $I^{2}=I$. In particular, if $I$ has an identity element, then $I$ is idempotent. Also, $I$ is said to be non-degenerate if for any $a \in I$ we have either $a I \neq 0$ or $I a \neq 0$.

It is not difficult to prove that if $I$ is either idempotent or non-degenerate, then $I$ is $(l, r)$-associative ([4], Proposition 2.5.).

Thus the following is immediate.

2.4. Corollary If $\alpha$ is a partial action of $G$ on an algebra $R$ such that each $D_{g}(g \in G)$ is either idempotent or non-degenerate, then the skew group ring $R \star_{\alpha} G$ is associative.

Note that if $R$ is a semiprime algebra, then any ideal of $R$ is nondegenerate. Then we have the following

2.5. Corollary If $R$ is a semiprime algebra, then $R \star_{\alpha} G$ is associative for any partial action $\alpha$ of a group $G$ on $R$.

An algebra with the property that $R \star_{\alpha} G$ is associative for any partial action $\alpha$ of a group $G$ on $R$ is called strongly associative.

The question of finding conditions under which a partial skew group ring is associative is an open problem. There are some results, of course. In particular we can quote [13]. In that paper the author studied strongly associative modular group algebras. But we think there are many other problems to investigate on the subject.

\section{Enveloping actions}

Natural examples of partial actions can be obtained by restricting a global action to an ideal.

3.1. Example Suppose that a group $G$ acts on an algebra $T$ by automorphisms $\beta_{g}: T \rightarrow T$ and let $R$ be an ideal of $T$. Assume that $D_{g}=R \cap \beta_{g}(R)$, for every $g \in G$. Define $\alpha_{g}$ as the restriction of $\beta_{g}$ to $D_{g^{-1}}$. Then it is easy to verify that $\alpha=\left\{\alpha_{g}: D_{g^{-1}} \rightarrow D_{g} \mid g \in G\right\}$ is a partial action of $G$ on $R$.

In the above case we say that $\alpha$ is the restriction of $\beta$ to $R$. Now we discuss conditions under which a given partial action can be obtained as the restriction of a global action.

If $G$ acts on $T$ by automorphisms and $R$ is an ideal of $T$, denote by $T^{\prime}$ the subalgebra of $T$ generated by $\cup_{g \in G} \beta_{g}(R)$. Since $T^{\prime}$ is invariant with respect to $\beta$, we see that $\alpha$ can be obtained also as a restriction of an action of $G$ on $T^{\prime}$. Thus in order to have uniqueness it is reasonable to require that $T^{\prime}=T$. In this case we say that $\alpha$ is an admissible restriction of $\beta$.

Now we define equivalent partial actions (see [4], Section 4): 
3.2. Definition Given two partial actions $\alpha=\left\{\alpha_{g}: D_{g^{-1}} \rightarrow D_{g} \mid g \in G\right\}$ and $\alpha^{\prime}=\left\{\alpha_{g}^{\prime}: D_{g^{-1}}^{\prime} \rightarrow D_{g}^{\prime} \mid g \in G\right\}$ of a group $G$ on algebras $R$ and $R^{\prime}$, respectively, we say that $\alpha$ and $\alpha^{\prime}$ are equivalent if there exists an algebra isomorphism $\varphi: R \rightarrow R^{\prime}$ such that for every $g \in G$ the following conditions hold:

(•) $\varphi\left(D_{g}\right)=D_{g}^{\prime}$;

$(\bullet \bullet) \alpha_{g}^{\prime} \circ \varphi(x)=\varphi \circ \alpha_{g}(x)$, for all $x \in D_{g^{-1}}$.

The definition of enveloping action given in [4], Definition 4.2., is the following:

3.3. Definition An action $\beta$ of a group $G$ on an algebra $T$ is said to be an enveloping action for the partial action $\alpha$ of $G$ on $R$ if $\alpha$ is equivalent to an admissible restriction of $\beta$ to an ideal of $T$.

In other words, the action $\beta$ of $G$ on $T$ is an enveloping action for $\alpha$ if there exists an algebra isomorphism $\varphi$ of $R$ onto an ideal of $T$ such that for all $g \in G$ the following three properties are satisfied:

(1) $\varphi\left(D_{g}\right)=\varphi(R) \cap \beta_{g}(\varphi(R))$;

(2) $\varphi \circ \alpha_{g}(x)=\beta_{g} \circ \varphi(x)$ for any $x \in D_{g^{-1}}$;

(3) $T$ is generated by $\cup_{g \in G} \beta_{g}(\varphi(R))$.

If the partial action $\alpha$ of $G$ on $R$ has an enveloping action $(T, \beta)$ we may assume, unless equivalence, that $R$ is an ideal of $T$ and $\beta$ is an action of $G$ on $T$ by automorphisms $\left\{\beta_{g} \mid g \in G\right\}$ such that $T$ is generated by $\cup_{g \in G} \beta_{g}(R)$, $D_{g}=R \cap \beta_{g}(R)$ and $\beta_{g}(x)=\alpha_{g}(x)$, for any $g \in G$ and $x \in D_{g^{-1}}$. It can easily be seen that in this case $T=\sum_{g \in G} \beta_{g}(R)$.

The question of whether a partial action possesses an enveloping action has been investigated when $R$ is a ring with identity element ([4], Theorem 4.5):

3.4. Theorem Let $R$ be a unital algebra. Then a partial action $\alpha$ of $G$ on $R$ admits an enveloping action $(T, \beta)$ if and only if each ideal $D_{g}$ has an identity element. Moreover, in this case the enveloping action is unique up to equivalence.

Note that an ideal $I$ of a ring $R$ has identity element if and only if $I$ is generated by a central idempotent of $R$. 
There is another paper in which the existence of enveloping actions is investigated (see [6]). However we do not want to include too many details here.

If $\alpha$ has an enveloping action, from Corollary 2.4 and Theorem 3.4 we have that $R \star_{\alpha} G$ is associative. However there is another reason showing that the existence of an enveloping action is quite important. In fact, we have the following:

3.5. Proposition If $\beta$ is an action of a group $G$ on an algebra $T$ which is an enveloping action for the partial action $\alpha$ of $G$ on $R$, then the partial skew group ring $R \star_{\alpha} G$ can be embedded into the skew group ring $T \star_{\beta} G$. In particular, $R \star_{\alpha} G$ is associative.

There is another kind of enveloping action for which Proposition 3.5 also holds. We will see this in Section 5.

\section{Elementary properties of partial actions and enveloping actions}

In a recent paper written by the author and J. Lazzarin [11] we study relations between the properties of $R$ and $T$, when $\alpha$ is a partial action of a group $G$ and $(T, \beta)$ is the enveloping action of $\alpha$. All the results of this section are contained in that paper.

First we note that since $T$ is a sum of the ideals $\beta_{g}(R), g \in G, T$ does not have an identity element, in general. To study when $T$ has an identity we need the following

4.1. Definition We say that $\alpha$ is of finite type if there exists a finite subset $\left\{g_{1}, \ldots, g_{n}\right\}$ of $G$ such that $\sum_{1 \leq i \leq n} D_{g g_{i}}=R$, for any $g \in G$.

It is clear that if $G$ is a finite group any partial action of $G$ on $R$ is of finite type. We have the following characterization (see [11], Proposition 1.2).

4.2. Proposition Assume that $\alpha$ is a partial action of $G$ on $R$ with enveloping action $(T, \beta)$. The following conditions are equivalent:

(i) $\alpha$ is of finite type;

(ii) There exist $g_{1}, \ldots, g_{n} \in G$ such that $T=\sum_{1 \leq i \leq n} \beta_{g_{i}}(R)$;

(iii) T has an identity element.

From the above proposition the following is easy to prove.

São Paulo J.Math.Sci. 3, 1 (2009), 95-107 
4.3. Corollary Assume that $R$ is a ring and $\alpha$ is a partial action of a group $G$ on $R$ with enveloping action $(T, \beta)$. Then $T$ is right (left) noetherian (artinian) if and only $R$ is right (left) noetherian (artinian) and $\alpha$ is of finite type.

4.4. Example $A$ partial action on an artinian ring which is not of finite type and $T$ is neither artinian nor noetherian.

Take $R=K e_{1} \oplus K e_{2}$, a direct sum of two copies of a field $K$, and $G$ an infinite cyclic group generated by $g$. We define a partial action of $G$ on $R$ by taking $D_{g^{0}}=R, \alpha_{g^{0}}=i d_{R}$, and $D_{g^{i}}=K e_{2}, \alpha_{g^{i}}=i d_{K e_{2}}$, for any $i \neq 0$. Then it is easy to see that this is in fact a partial action and the enveloping action $\beta$ of $\alpha$ is defined on the ring $T$ which is the direct sum of the copies $K e_{i}$ of $K, i \in \mathbf{Z}$, by $\beta_{g}\left(e_{2}\right)=e_{2}, \beta_{g}\left(e_{1}\right)=e_{3}$ and $\beta_{g}\left(e_{j}\right)=e_{j+1}$ for any $j \neq 1,2$. It is clear that $\alpha$ is not of finite type.

An interesting result proved in ([4], Theorem 4.2) is the following

4.5. Theorem Assume that $\alpha$ is a partial action of $G$ on $R$ having an enveloping action $(T, \beta)$, where $T$ has an identity. Then the partial skew group ring $R \star_{\alpha} G$ and the skew group ring $T \star_{\beta} G$ are Morita equivalent.

The following is an immediate consequence of Proposition 4.2 and Theorem 4.5.

4.6. Corollary If $\alpha$ is a partial action of finite type of $G$ on $R$, the partial skew group ring $R \star_{\alpha} G$ and the skew group ring $T \star_{\beta} G$ are Morita equivalent.

It is well-known that if $T$ is left (right) noetherian (artinian) and $G$ is finite, then $T \star_{\beta} G$ is left (right) noetherian (artinian). Thus the following is immediate from Corollary 4.6.

4.7. Proposition If $R$ is left (right) noetherian (artinian) and $G$ is finite, then $R \star_{\alpha} G$ is left (right) noetherian (artinian).

The following is an easy consequence of the above results.

4.8. Corollary Assume that $\alpha$ is a partial action of a group $G$ on $R$ with enveloping action $(T, \beta)$. Then $T$ is semisimple if and only if $R$ is semisimple and $\alpha$ is of finite type.

To study relations between the properties of $R$ and $T$ we use the fact that $T$ is a sum of ideals all of which are isomorphic to $R$.

Recall that a ring $S$ is said to be a ring with local units if for any finitely many $s_{1}, \ldots, s_{k} \in S$ there exists an idempotent $e \in S$ with $\left\{s_{1}, \ldots, s_{k}\right\} \subseteq e S e$ ([19], Ch. 10). Also, $S$ is a right (left) $s$-unital ring if for any $x \in \bar{S}$ one 
has $x \in x S(x \in S x)$ [6]. It is clear that a ring with local units is a right and left $s$-unital ring.

Since any finite subset of $T$ is contained in a subring which is a finite sum of the ideals $\beta_{g}(R), g \in G$, the following is an easy consequence.

4.9. Corollary Assume that $\alpha$ is a partial action of a group $G$ on a ring $R$ with enveloping action $(T, \beta)$. Then $T$ is a ring with local units.

Recall that a radical $\gamma$ is said to be hereditary if for any ring $R$ and ideal $I$ of $R$ we have $\gamma(I)=\gamma(R) \cap I$ ([3], p. 125).

4.10. Proposition Assume that $\alpha$ is a partial action of a group $G$ on $R$ with enveloping action $(T, \beta)$. If $\gamma$ is a hereditary radical, then $R$ is $\gamma$-semisimple if and only if $T$ is $\gamma$-semisimple.

As a particular case of Proposition 4.10 we immediately have

4.11. Corollary Under the above assumption, $R$ is semiprime (semiprimitive) if and only if $T$ is semiprime (semiprimitive).

If $R$ is prime, then $R$ does not have nonzero central idempotents. Thus any ideal $D_{g}$ must be either $R$ or zero (this possibility is not excluded in [4] for partial actions with enveloping actions), so the partial action is not necessarily a global one, in general. However, if $T$ is prime, then clearly $T=R$ and the partial action is a global action on $R$.

Recall that $R$ is said to be von Neumann regular if for any $a \in R$ there exists $b \in R$ such that $a b a=a$. For equivalent conditions of the definition see Theorem 4.23 of [14].

4.12. Proposition Assume that $\alpha$ is a partial action of $G$ on $R$ with enveloping action $(T, \beta)$. Then $R$ is von Neumann regular if and only if $T$ is von Neumann regular.

If $\alpha$ is a partial action of finite type of $G$ on $R$ with enveloping action $(T, \beta)$, there exist a smallest integer $n \geq 1$ and $g_{1}, \ldots, g_{n} \in G$ such that $T=\sum_{1 \leq i \leq n} \beta_{g_{i}}(R)$. In this case the order of $\alpha$ is defined as $n$.

We denote by $\operatorname{udim}_{S} S$ the uniform dimension of $S$ as left $S$-module.

4.13. Proposition Let $R$ be a ring and $\alpha$ a partial action of finite type of $G$ on $R$ with enveloping action $(T, \beta)$. Then udim $_{R} R \leq \operatorname{udim}_{T} T \leq$ $o(\alpha)$ udim $_{R} R$, where $o(\alpha)$ is the order of $\alpha$. In particular, if $G$ is a finite group of order $|G|$ we have udim $_{T} T \leq|G|$ udim $_{R} R$.

Denote by $\mathcal{Z}(S)$ the left singular ideal of $S$. 
4.14. Lemma Assume that $S=\sum_{i \in \mathcal{I}} B_{i}$, where $B_{i}$ is an ideal of $S$ which has identity $1_{i}$ as a ring, for any $i$. Then we have $\mathcal{Z}\left(B_{i}\right)=\mathcal{Z}(S) \cap B_{i}$, for any $i$.

The following is an immediate consequence of the above.

4.15. Corollary Let $R$ be a ring and $\alpha$ a partial action of $G$ on $R$ with enveloping action $(T, \beta)$. Then $R$ is left non-singular if and only if $T$ is left non-singular.

Putting together Propositions 4.11, 4.13 and 4.15 we have the following

4.16. Corollary Assume that $R$ is a ring and $\alpha$ is a partial action of $G$ on $R$ with enveloping action $(T, \beta)$. Then $T$ is a semiprime left Goldie ring if and only if $R$ is semiprime left Goldie and $\alpha$ is of finite type.

Finally the following result is easy to show,

4.17. Proposition Let $R$ be a ring, $\alpha$ a partial action of a group $G$ on $R$ with enveloping action $(T, \beta)$ and $m$ a positive integer. Then

(i) $R$ do not have $m$-additive torsion if and only if $T$ do not have $m$ additive torsion.

(ii) If, in addition, $T$ has an identity element, then $m$ is invertible in $R$ if and only if $m$ is invertible in $T$.

\section{Partial actions on semiprime rings}

As we said above there is another kind of enveloping action for which Proposition 3.5 also holds. The following definition was given by the author [10].

5.1. Definition An action $\beta=\left\{\beta_{g} \mid g \in G\right\}$ of a group $G$ on an algebra $T$ is said to be a weak enveloping action for the partial action $\alpha$ of $G$ on $R$ if $R$ is a subalgebra of $T$ (not necessarily a unital subalgebra) and for any $\left.g \in G \beta_{g}\right|_{D_{g^{-1}}}=\alpha_{g}$.

It is clear, as in Proposition 3.5, that if a partial action admits a weak enveloping action $(T, \beta)$, then the partial skew group ring $R \star_{\alpha} G$ is a subring of the skew group ring $T \star_{\beta} G$ and then $R \star_{\alpha} G$ is associative.

It remains as an open problem to determining conditions under which a partial action admits a weak enveloping action. In [10] we proved the following:

5.2. Theorem Assume that $R$ is a semiprime algebra and $\alpha$ is a partial action of a group $G$ on $R$. Then $\alpha$ possesses a weak enveloping action. 
Note that in [10] we assumed that any of the ideals $D_{g}, g \in G$, is nonzero. However, this assumption is actually not necessary to prove the result.

From the above result it also follows that if $R$ is semiprime, then $R \star_{\alpha} G$ is associative. This gives another proof of a result which is already known.

The proof of the main result in [10] uses the Martindale ring of right (left) quotients $Q$ of the semiprime ring $R$. First it is proved that a partial action $\alpha$ on $R$ can be extended to a partial action $\alpha^{*}$ of $Q$. The ideals $D_{g}^{*}$ of the partial action $\alpha^{*}$ are closed ideals of $Q$ and so they are generated by central idempotents. Thus this extension of $\alpha$ to $Q$ admits an enveloping action and so the results of [4] can be applied. Hence the week enveloping action of $\alpha$ is the enveloping action of $\left(Q, \alpha^{*}\right)$.

\section{Properties of partial skew group rings}

For the remainder of the paper we assume that the partial action $\alpha$ has an enveloping action $(T, \beta)$. The results in the following are proved in [11].

We begin the section with two versions of Maschke's Theorem.

First we recall some notions from [5]. For a partial action $\alpha$ of a group $G$ on $R$, the invariant subring $R^{\alpha}$ has been defined as follows:

$$
R^{\alpha}=\left\{a \in R \mid \alpha_{g}\left(a 1_{g^{-1}}\right)=a 1_{g} \text {, for any } g \in G\right\} .
$$

When $G$ is finite, also the trace map $t r_{\alpha}: R \rightarrow R^{\alpha}$ has been defined in [5] by

$$
t r_{\alpha}(a)=\sum_{g \in G} \alpha_{g}\left(a 1_{g^{-1}}\right)
$$

for any $a \in R$. It is proved that $\operatorname{tr}_{\alpha}: R \rightarrow R^{\alpha}$ is a (left and right) $R^{\alpha}$-linear map.

The following is an easy consequence of the Morita equivalence between $R \star_{\alpha} G$ and $T \star_{\beta} G$.

6.1. Theorem Let $R$ be a ring and $\alpha$ a partial action of a finite group $G$ on $R$. If $R$ is semisimple and $|G|$ is invertible in $R$, then $R \star_{\alpha} G$ is semisimple.

The proof of the second version follows the lines of Theorem 0.1 of [15].

6.2. Theorem Let $\alpha$ be a partial action of a finite group $G$ on $R, V$ a left $R \star_{\alpha} G$-module and $W$ a submodule of $V$. If $\operatorname{tr}_{\alpha}\left(1_{R}\right)$ is invertible in $R$ and $W$ is a direct summand of $V$ as an $R$-module, then $W$ is a direct summand of $V$ as an $R \star_{\alpha} G$-module.

Theorem 6.2 immediately implies: 
6.3. Corollary If $R$ is semisimple, $\alpha$ is a partial action of a finite group $G$ on $R$ and $\operatorname{tr}_{\alpha}\left(1_{R}\right)$ is invertible in $R$, then $R \star_{\alpha} G$ is semisimple.

The example below shows that the assumptions of Theorems 6.1 and 6.2 are independent.

6.4. Example Take $R=K e_{1} \oplus K e_{2} \oplus K e_{3}$, where $K$ is a ring and $e_{1}, e_{2}, e_{3}$ are orthogonal central idempotents of $R$. Let $G$ be the cyclic group of order 4 with generator $g$ and define a partial action of $G$ on $R$ by: $\alpha_{1}=i d_{R}$,

$$
\begin{aligned}
& \alpha_{g}: K e_{2} \oplus K e_{3} \rightarrow K e_{1} \oplus K e_{2}, \alpha_{g}\left(e_{2}\right)=e_{1} \text { and } \alpha_{g}\left(e_{3}\right)=e_{2} ; \\
& \alpha_{g^{2}}: K e_{1} \oplus K e_{3} \rightarrow K e_{1} \oplus K e_{3}, \alpha_{g^{2}}\left(e_{1}\right)=e_{3} \text { and } \alpha_{g^{2}}\left(e_{3}\right)=e_{1} ; \\
& \alpha_{g^{3}}: K e_{1} \oplus K e_{2} \rightarrow K e_{2} \oplus K e_{3}, \alpha_{g^{3}}\left(e_{1}\right)=e_{2} \text { and } \alpha_{g^{3}}\left(e_{2}\right)=e_{3} .
\end{aligned}
$$

Take $K$ equal to the ring of integers modulo 15 . Then $|G|=41_{R}$ is invertible in $R$ and $\operatorname{tr}_{\alpha}\left(1_{R}\right)=31_{R}$ is not invertible.

Now take $K$ equal to the ring of integers modulo 2 . Then $|G|$ is not invertible in $R$ and $\operatorname{tr}_{\alpha}\left(1_{R}\right)$ is invertible. Note that in both cases $R$ is a semisimple ring.

Now we consider von Neumann regularity. In [11] we proved the following 6.5. Theorem Assume that $R$ is a von Neumann regular ring, $\alpha$ is a partial action of a finite group $G$ on $R$ and $|G|$ is invertible in $R$. Then $R \star_{\alpha} G$ is von Neumann regular.

We have a similar result using the assumption that $\operatorname{tr}_{\alpha}\left(1_{R}\right)$ is invertible in $R$. The proof is inspired by the one in ([2], Theorem 1.3) for skew group rings.

6.6. Lemma Let $R$ be a von Neumann regular ring and $\alpha$ a partial action of a finite group $G$ on $R$. If $I$ is a left principal ideal of $R \star_{\alpha} G$, then $I$ is an $R$-direct summand of $R \star_{\alpha} G$.

Using the above lemma and Theorem 6.2 we obtain the following

6.7. Theorem Assume that $R$ is a von Neumann regular ring and $\alpha$ is a partial action of a finite group $G$ on $R$ where $\operatorname{tr}_{\alpha}\left(1_{R}\right)$ is invertible in $R$. Then $R \star_{\alpha} G$ is von Neumann regular.

\section{Prime and primitive ideals, the prime radical and the Ja- cobson radical.}

The following was proved in [11] using the Morita context constructed in [4], Section 5. 
7.1. Theorem There is a one-to-one correspondence, via contraction, between the set of all prime ideals of $R \star_{\alpha} G$ and the set of all prime ideals of $T \star_{\beta} G$.

From the above theorem we immediately have

7.2. Corollary $\operatorname{rad}\left(R \star_{\alpha} G\right)=\operatorname{rad}\left(T \star_{\beta} G\right) \cap\left(R \star_{\alpha} G\right)$.

Another consequence which follows directly from ([4], Theorem 5.4) is the following

7.3. Proposition Assume that $G$ is a finite group and $R$ is semiprime without $|G|$-torsion. Then $R \star_{\alpha} G$ is semiprime.

Concerning primitive ideals we have the following. Assume that $P \triangleleft R \star_{\alpha}$ $G$ and $P^{\prime} \triangleleft T \star_{\beta} G$ are corresponding prime ideals, i.e., $P^{\prime} \cap\left(R \star_{\alpha} G\right)=P$.

7.4. Lemma $P$ is left (right) primitive if and only if $P^{\prime}$ is left (right) primitive.

Denote by $J(A)$ the Jacobson radical of the ring $A$. The following easily follows.

7.5. Corollary $J\left(R \star_{\alpha} G\right)=J\left(T \star_{\beta} G\right) \cap\left(R \star_{\alpha} G\right)$.

Now we assume that $G$ is finite. The following result extends to partial skew group rings a result which is known for skew group rings (Theorem 4.2 of [17]):

7.6. Theorem Assume that $\alpha$ is a partial action of a finite group $G$ on the ring $R$. Then $J\left(R \star_{\alpha} G\right)^{|G|} \subseteq J(R) \star_{\alpha} G \subseteq J\left(R \star_{\alpha} G\right)$.

In addition, if $|G|$ is invertible in $R$, then $J\left(R \star_{\alpha} G\right)=J(R) \star_{\alpha} G$.

7.7. Corollary Assume that $R$ is a ring and $\alpha$ is a partial action of a finite group $G$ on $R$. Then

(i) $J\left(R \star_{\alpha} G\right) \cap R=J(R)$.

(ii) $J\left(R \star_{\alpha} G\right)$ is nilpotent if and only if $J(R)$ is nilpotent.

The equality $J\left(R \star_{\alpha} G\right)=J(R) \star_{\alpha} G$, proved in Theorem 7.6 when $|G|$ is invertible in $R$ also holds under the assumption $\operatorname{tr}_{\alpha}\left(1_{R}\right) \in U(R)$ [11].

7.8. Proposition Assume that $R$ is a ring and $\alpha$ is a partial action of a finite group $G$ on $R$ with $\operatorname{tr}_{\alpha}\left(1_{R}\right) \in U(R)$. Then $J\left(R \star_{\alpha} G\right)=J(R) \star_{\alpha} G$.

We finish the paper with the following

7.9. Corollary Assume that $R$ is a ring and $\alpha$ is a partial action of a finite group $G$ on $R$. If $R$ is Jacobson semisimple and at least one of the elements $\operatorname{tr}_{\alpha}\left(1_{R}\right)$ or $|G|$ is invertible in $R$, then $R \star_{\alpha} G$ is also Jacobson semisimple. 


\section{References}

[1] Abadie, F.; Enveloping actions and Takei duality for partial actions, J. Funct. Anal. 197 (2003), 14-67.

[2] Alfaro, R., Ara, P., del Rio, A.; Regular skew group rings, J. Austral Math. Soc. (Series A) 58 (1995), 167-182.

[3] Divinsky, N. J.; Rings and Radicals, Univ. of Toronto Press (1965).

[4] Dokuchaev M., Exel, R; Associativity of crossed products by partial actions, enveloping actions and partial representations, Trans. Amer. Math. Soc. 357 (2005), 1931-1952.

[5] Dokuchaev, M., Ferrero, M., Paques, A.; Partial actions and Galois theory; J. Pure Apll. Algebra 208 (2007), 77-87.

[6] Dokuchaev, M., del Rio, A., Simón, J. J.; Globalizations of partial actions on nonunital rings; Proc. Amer. Math. Soc. 135 (2007), 343-352.

[7] R. Exel, Twisted partial actions: a classification of regular $C^{*}$-algebraic bundles, Proc. London Math. Soc. 74 (3) (1997), 417 - 443.

[8] Exel, R; Partial actions of groups and actions of semigroups, Proc. Amer. Math. Soc. 126 (12) (1998), 3481-3494.

[9] Exel, R., Laca, M., Quigg, J. C.; Partial dynamical systems and $C^{*}$-algebras generated by partial isometries, J. Operator Theory 47 (1) (2002), 169-186.

[10] Ferrero, M.; Partial actions of groups on semiprime rings; Groups, Rings and Group Rings, Lect. Notes in Pure Apll. Math. 248, Chapman and Hall (2006), 155-162.

[11] Ferrero, M., Lazzarin, J.; Partial actions and partial skew group rings; J. Algebra 319(2008), 5247-5264.

[12] Fillmore, P. A.; A user's Guide to Operator Algebras, Willey -Interscience, 1996.

[13] Gonçalves Lopes, J.; Strongly associative modular group algebras, Comm. Algebra 36(2008), 478-492.

[14] Lam, T. Y.; A First Course on Noncommutative Rings, 2nd ed.,Graduate text in Math. 131, Springer, New York, Berlin, Heidelberg, 2001.

[15] Montgomery, S.; Fixed Rings of Finite Automorphism Groups of Associtive Rings; Lect. Notes in Math. 818, Springer, Berlin, 1980.

[16] McClanahan, K.; K-theory for partial crossed products by discrete groups, J. Funct. Anal. 130 (1) (1995), 77 - 117.

[17] Passman, D. S.; Infinite Crossed Products, Academic Press, San Diego, 1989.

[18] Quigg, J. C., Raeburn, I.; Characterizations of crossed Products by Partial Actions, J. Operator Theory 37 (1997), 311-340.

[19] Wisbauer, R.; Foundations of Modules and Rings, Gordon and Breach, London 1991. 